\title{
A MONOTONE METHOD FOR FOURTH ORDER PERIODIC BOUNDARY VALUE PROBLEMS AND PERIODIC SOLUTIONS OF FUNCTIONAL DIFFERENTIAL EQUATIONS*
}

\author{
WENJIE ZUO ${ }^{\dagger}$, DAQING JIANG ${ }^{\ddagger}$, DONAL O’REGAN§ , AND R. P. AGARWAL
}

\begin{abstract}
In this paper, we show that the monotone iterative technique yields two monotone sequences that converge uniformly to extremal solutions of fourth order periodic boundary value problems and periodic solutions of functional differential equations.
\end{abstract}

Key words. Periodic boundary value problem, Periodic solution, Existence, Upper and lower solutions, Monotone iterative technique

AMS subject classifications. 34K06, 34K10

1. Introduction. The method of upper and lower solutions coupled with the monotone iterative has been applied successfully to obtain existence results and approximation of solutions for periodic boundary value problems for first order and second order ordinary differential equations (see [1] and references therein).

Some attempts have been made to extend these techniques to study periodic boundary value problems and periodic solutions of first order and second order functional differential equations (FDEs). For example, see [2-6, 10] and references therein. As far as the authors know, the method of upper and lower solutions coupled with the monotone iterative technique has rarely been applied in the study of periodic boundary value problems and periodic solutions of higher order functional differential equations.

In this paper, we consider the fourth order periodic boundary value problem(PBVP)

$$
\left\{\begin{array}{l}
y^{(4)}(t)=f(t, y(t), y(w(t))), \quad t \in I=[0, T] \\
y^{(i)}(0)=y^{(i)}(T), \quad i=0,1,2,3
\end{array}\right.
$$

here $f \in C\left(I \times R^{2}, R\right), w \in C(I,[a, b])$, and $a, b$ are constants such that $[0, T] \subset$ $[a, b], T>0$.

We also consider in this paper $T$-periodic solutions of the functional differential equation(FDE)

$$
y^{(4)}(t)=f(t, y(t), y(w(t))), \quad t \in R
$$

here $f \in C\left(R^{3}, R\right), f(t, u, v)=f(t+T, u, v), T>0, w(t)=t-\tau(t), \quad \tau \in$ $C(R, R), \tau(t)=\tau(t+T)$.

${ }^{*}$ Received October 29, 2004; accepted for publication March 17, 2005. The work was supported by NNSF of China.

†School of Mathematical Sciences, Jilin University, Changchun, Jilin 130021, P. R. China.

${ }_{\ddagger}^{\ddagger}$ Department of Mathematics, Northeast Normal University, Changchun, Jilin 130024, P. R. China (daqingjiang@vip.163.com).

$\S$ Department of Mathematics, National University of Ireland, Galway, Ireland (donal.oregan@ nuigalway.ie).

TDepartment of Mathematical Science, Florida Institute of Technology, Melbourne, Florida 329016975, USA (agarwal@fit.edu). 
Note that (1.1) and (1.2) include ordinary, retarded and advanced differential equations.

In [7] and [8], Cabada and Lois obtained a maximum principle for the fourthorder operator $L_{4, \alpha} u=u^{(4)}-\alpha u$ with periodic boundary conditions, and they proved the existence of solutions and the validity of the monotone method in the presence of lower and upper solutions for the periodic boundary problem

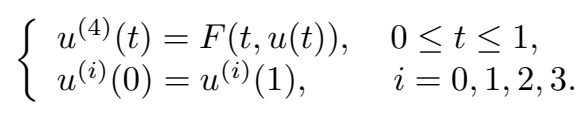

Recently, Jiang and Kong [9] studied the positivity of the Green's function for $-u^{(4)}+$ $\alpha u=0$ with periodic boundary conditions, and they established existence criteria for multiple positive solutions using a fixed point theorem in cones. Note other higher order results can be found in [11-15].

In this paper, Section 2 is devoted to the maximum principle, which is the key to developing the monotone technique. Section 3 discusses the monotone method for (1.1) and Section 4 discusses the monotone method for (1.2).

2. Maximum principle. To prove the validity of the monotone iterative technique, we shall use a maximum principle. In the proof of our maximum principle below, we will use a lemma of Alberto Cabada [8].

Lemma 2.1. ${ }^{[8]}$ If the linear problem

$$
\begin{aligned}
z^{(n)}(t)+M z(t) & =0, \\
z^{(i)}(a)-z^{(i)}(b) & =0, \quad i=0, \cdots, n-2, \\
z^{(n-1)}(a)-z^{(n-1)}(b) & =1,
\end{aligned}
$$

has a unique solution $r \in C^{\infty}[a, b]$, the problem

$$
\begin{aligned}
& u^{(n)}(t)+M u(t)=\sigma(t) \in L^{1}(I), \\
& u^{(i)}(a)-u^{(i)}(b)=\lambda_{i}, \quad i=0, \cdots, n-1,
\end{aligned}
$$

has a unique solution u given by the expression

$$
u(t)=\int_{a}^{b} G_{n}(t, s) \sigma(s) d s+\sum_{i=0}^{n-1} r^{(i)}(t) \lambda_{n-1-i},
$$

where

$$
G_{n}(t, s)= \begin{cases}r(a+t-s), & a \leq s \leq t \leq b \\ r(b+t-s), & a \leq t \leq s \leq b\end{cases}
$$

Next, we define

$$
G(t, m)=G(|t-s|, m):= \begin{cases}\frac{f(t-s)+g(t-s)}{4 m^{3}\left(e^{\frac{m T}{2}}-e^{-\frac{m T}{2}}\right)^{2}(\cos m T-1)}, & 0 \leq s \leq t \leq T, \\ \frac{f(T+t-s)+g(T+t-s)}{4 m^{3}\left(e^{\frac{m T}{2}}-e^{-\frac{m T}{2}}\right)^{2}(\cos m T-1)}, & 0 \leq t \leq s \leq T,\end{cases}
$$


where

$$
f(t):=\left(e^{\frac{m T}{2}}-e^{-\frac{m T}{2}}\right)^{2}(\sin m t+\sin m(T-t)),
$$

and

$$
g(t):=\left(e^{m t}-e^{-m t}+e^{m(T-t)}-e^{-m(T-t)}\right)(1-\cos m T) .
$$

By similar arguments as in [9], we obtain the following result.

LEмma 2.2. If $m \in\left(0, \frac{2 \pi}{T}\right)$, then the function $G(t, m)$ in the interval $[0, T]$ attains its maximum at $t=0$ and its minimum at $t=\frac{T}{2}$.

From Lemma 2.2, the greatest value of $m$ for which $G(t, m)$ is negative in $[0, T]$ will be the smallest positive zero of the expression

$$
f(0)+g(0)=\left(e^{m T}-e^{-m T}\right)(1-\cos m T)+\left(e^{\frac{m T}{2}}-e^{-\frac{m T}{2}}\right)^{2} \sin m T .
$$

This expression is zero if and only if either $m=\frac{2 \pi n}{T}, n \in N$ or

$$
\tan \frac{m T}{2}=-\tanh \frac{m T}{2} .
$$

The smallest positive root of (2.4) will be denoted by $k$. This is the unique root in $\left(0, \frac{2 \pi}{T}\right)$.

Let $m \in(0, k) \subset\left(0, \frac{2 \pi}{T}\right)$. Then $G(0, m)<0$.

REMARK. When $T=2 \pi$, the smallest positive root $k$ of (2.4) takes a value of 0.7528094 with an error of $\pm 10^{-7}$ (see[8, 9]).

Theorem 2.1. Let $y \in E=C([a, b], R) \cap C^{4}([0, T], R)$ and $M=m^{4}, m \in$ $(0, k), N>0$ such that

(i) $\quad y^{(4)}(t)-M y(t)-N y(w(t)) \geq 0, \quad t \in I$,

(ii) $y^{(i)}(0)=y^{(i)}(T), \quad i=0,1,2$, $y^{(3)}(0) \geq y^{(3)}(T)$

(iii) $y(0)=y(t), t \in[a, 0] \bigcup[T, b]$,

and

(iv) $\frac{N}{M}\left(\frac{1}{\delta}-1\right)<1$, here $\delta=\frac{\left(e^{m T}-1\right) \cos \frac{m T}{2}+\left(e^{m T}+1\right) \sin \frac{m T}{2}}{e^{m T}+2 e^{\frac{m T}{2}} \sin \frac{m T}{2}-1}$,

where $k$ is the smallest positive root of the equation $\tan \frac{m T}{2}=-\tanh \frac{m T}{2}$, and $w \in$ $C(I,[a, b])$.

Then $y(t) \leq 0, \quad \forall t \in[a, b]$.

Proof. There exists $\xi \in[0, T]$ such that

$$
y(\xi)=\min _{t \in[0, T]} y(t)=\min _{t \in[a, b]} y(t),
$$

and so

$$
\begin{gathered}
y^{(4)}(t)-M y(t)-N y(\xi) \geq 0, \quad t \in I, \\
y^{(i)}(0)=y^{(i)}(T), \quad i=0,1,2, \\
y^{(3)}(0) \geq y^{(3)}(T) .
\end{gathered}
$$


Let $\sigma(t)=y^{(4)}(t)-M y(t)-N y(\xi) \geq 0$ and $\lambda=y^{(3)}(0)-y^{(3)}(T) \geq 0$.

Then we have

$$
\begin{gathered}
y^{(4)}(t)-M y(t)-N y(\xi)=\sigma(t), \quad t \in I, \\
y^{(i)}(0)=y^{(i)}(T), \quad i=0,1,2, \\
y^{(3)}(0)=y^{(3)}(T)+\lambda .
\end{gathered}
$$

Let $u(t)=y(t)+\frac{N}{M} y(\xi)$, and so

$$
\begin{aligned}
& u^{(4)}(t)-M u(t)=\sigma(t), \quad t \in I, \\
& u^{(i)}(0)=u^{(i)}(T), \quad i=0,1,2, \\
& u^{(3)}(0)=u^{(3)}(T)+\lambda .
\end{aligned}
$$

Let $r(t)$ be the unique solution $\left(r \in C^{\infty}[0, T]\right)$ to the problem

$$
\begin{aligned}
& z^{(4)}(t)-M z(t)=0 \\
& z^{(i)}(0)=z^{(i)}(T), \quad i=0,1,2, \\
& z^{(3)}(0)=z^{(3)}(T)+1
\end{aligned}
$$

From Lemma 2.1, we obtain

$$
u(t)= \begin{cases}\int_{0}^{T} G(0, s, m) \sigma(s) d s+\lambda r(0), & t \in[a, 0] \bigcup[T, b], \\ \int_{0}^{T} G(t, s, m) \sigma(s) d s+\lambda r(t), & t \in[0, T],\end{cases}
$$

where $m=\sqrt[4]{M}$, and $G(t, s, m), f(t), g(t)$ are defined above Lemma 2.2 and

$$
r(t)=\frac{f(t)+g(t)}{4 m^{3}\left(e^{\frac{m T}{2}}-e^{-\frac{m T}{2}}\right)^{2}(\cos m T-1)} .
$$

From Lemma 2.2, a direct calculation shows that

$$
r\left(\frac{T}{2}\right) \leq G(t, s, m) \leq r(0)<0
$$

here

$$
r\left(\frac{T}{2}\right)=\frac{e^{m T}+2 e^{\frac{m T}{2}} \sin \frac{m T}{2}-1}{4 m^{3}\left(1-e^{m T}\right) \sin \frac{m T}{2}}
$$

and

$$
r(0)=\frac{\left(e^{m T}-1\right) \cos \frac{m T}{2}+\left(e^{m T}+1\right) \sin \frac{m T}{2}}{4 m^{3}\left(1-e^{m T}\right) \sin \frac{m T}{2}}<0 .
$$


From (2.5) and (2.7) we have

$$
r\left(\frac{T}{2}\right)\left(\int_{0}^{T} \sigma(s) d s+\lambda\right) \leq u(t) \leq r(0)\left(\int_{0}^{T} \sigma(s) d s+\lambda\right), \quad \forall t \in[a, b] .
$$

Since $u(t)=y(t)+\frac{N}{M} y(\xi)$, we have from (2.8) that

$$
y(\xi)=\frac{M}{M+N} u(\xi) \geq \frac{M}{M+N} r\left(\frac{T}{2}\right)\left(\int_{0}^{T} \sigma(s) d s+\lambda\right) .
$$

On the other hand, from (2.8) we also have

$$
\begin{aligned}
y(t) & =u(t)-\frac{N}{M} y(\xi) \\
& \leq r(0)\left(\int_{0}^{T} \sigma(s) d s+\lambda\right)-\frac{N}{M} y(\xi) \\
& \leq\left(\int_{0}^{T} \sigma(s) d s+\lambda\right)\left(r(0)-\frac{N}{M+N} r\left(\frac{T}{2}\right)\right) \\
& =\left(\int_{0}^{T} \sigma(s) d s+\lambda\right) r\left(\frac{T}{2}\right)\left(\delta-\frac{N}{M+N}\right) ;
\end{aligned}
$$

here $\delta=\frac{r(0)}{r\left(\frac{T}{2}\right)}=\frac{\left(e^{m T}-1\right) \cos \frac{m T}{2}+\left(e^{m T}+1\right) \sin \frac{m T}{2}}{e^{m T}+2 e^{\frac{m T}{2}} \sin \frac{m T}{2}-1}$. Now from assumption (iv), we obtain $\frac{N}{M+N}<\delta$. Thus $y(t) \leq 0, \forall t \in[a, b]$.

In the same way as in Theorem 2.1, we obtain the following result.

Theorem 2.2. Let $y \in X=\left\{y \in C^{4}(R, R): y(t)=y(t+T)\right\}$ and $M=m^{4}, m \in$ $(0, k), N>0$ such that

(i) $\quad y^{(4)}(t)-M y(t)-N y(w(t)) \geq 0, \quad t \in R$,

(ii) $\frac{N}{M}\left(\frac{1}{\delta}-1\right) \leq 1$, here $\delta=\frac{\left(e^{m T}-1\right) \cos \frac{m T}{2}+\left(e^{m T}+1\right) \sin \frac{m T}{2}}{e^{m T}+2 e^{\frac{m T}{2}} \sin \frac{m T}{2}-1}$,

where $k$ is defined in Theorem 2.1 and $w(t)=t-\tau(t), \quad \tau \in C(R, R), \tau(t)=\tau(t+$ $T), \quad T>0$. Then $y(t) \leq 0, \quad \forall t \in R$.

3. Monotone method for PBVPs of fourth order FDE. In order to develop the monotone iterative technique for (1.1), we shall first consider the following PBVP

$$
\left\{\begin{array}{l}
y^{(4)}(t)-M y(t)-N y(w(t))=\sigma(t), \quad t \in I, \\
y^{(i)}(0)=y^{(i)}(T), \quad i=0,1,2,3 \\
y(t)=y(0), \quad t \in[a, 0] \bigcup[T, b]
\end{array}\right.
$$

where $\sigma \in C(I, R)$ and $w \in C(I,[a, b])$.

Let

$$
E^{*}=\{y \in E: y(t)=y(0), \quad \forall t \in[a, 0] \bigcup[T, b]\},
$$

where $E$ is defined as in Section 2. Endow $E^{*}$ with the norm

$$
\|y\|_{4}=\max _{t \in[a, b]}|y(t)|+\max _{t \in[0, T]}\left(\sum_{i=1}^{4}\left|y^{(i)}(t)\right|\right)
$$


for $y \in E^{*}$. Then $E^{*}$ is a Banach space.

A function $\alpha \in E^{*}$ is said to be a lower solution to (3.1), if it satisfies

$$
\left\{\begin{array}{l}
\alpha^{(4)}(t)-M \alpha(t)-N \alpha(w(t)) \geq \sigma(t), \quad t \in I, \\
\alpha^{(i)}(0)=\alpha^{(i)}(T), \quad i=0,1,2 \\
\alpha^{(3)}(0) \geq \alpha^{(3)}(T) .
\end{array}\right.
$$

An upper solution $\beta \in E^{*}$ for (3.1) is defined analogously by reversing the inequalities of above. A function $y \in E^{*}$ is said to be a solution to (3.1), if it is both an upper solution and a lower solution to (3.1).

For $\alpha, \beta \in E^{*}$, we shall write $\alpha \leq \beta$ if $\alpha(t) \leq \beta(t)$ for $\forall t \in[a, b]$. In such a case, we shall denote

$$
[\alpha, \beta]=\left\{y \in E^{*}: \alpha \leq y \leq \beta\right\}
$$

THEOREM 3.1. Suppose that there exists a lower solution $\alpha$ and an upper solution $\beta$ of (3.1) such that $\alpha \leq \beta$, and assume that $M=m^{4}, m \in(0, k), N>0$ satisfies condition (iv) of Theorem 2.1 (here $k$ is defined in Theorem 2.1). Then (3.1) has a unique solution $y \in[\alpha, \beta]$.

Proof. Consider the PBVP

$$
\left\{\begin{array}{l}
y^{(4)}(t)-M y(t)=N p(t, y(w(t)))+\sigma(t), \quad t \in I=[0, T] \\
y^{(i)}(0)=y^{(i)}(T), \quad i=0,1,2,3 \\
y(t)=y(0), \quad t \in[a, 0] \bigcup[T, b]
\end{array}\right.
$$

where

$$
p(t, x)= \begin{cases}\alpha(t), & \text { if } x<\alpha(t) \\ x, & \text { if } \alpha(t) \leq x \leq \beta(t) \\ \beta(t), & \text { if } x>\beta(t)\end{cases}
$$

Let us define an operator $\Phi: E^{*} \rightarrow E^{*}$ by

$$
(\Phi y)(t)= \begin{cases}\int_{0}^{T} G(t, s, m)[N p(s, y(w(s)))+\sigma(s)] d s, & t \in I=[0, T], \\ \int_{0}^{T} G(0, s, m)[N p(s, y(w(s)))+\sigma(s)] d s, & t \in[a, 0] \bigcup[T, b],\end{cases}
$$

where $G(t, s, m)$ is defined in the proof of Theorem 2.1. A standard argument shows that $\Phi: E^{*} \rightarrow E^{*}$ is continuous and compact (note since $N p(t, y(w(t)))+\sigma(t)$ is bounded on $I$, then $\Phi$ is bounded in $E^{*}$ ). The existence of a fixed point $y$ for the operator $\Phi$ follows now from the Schauder fixed point theorem. That means $(3.1)^{*}$ has a solution $y \in E^{*}$.

Now we will show that $y \in[\alpha, \beta]$. First, we prove that $y \geq \alpha$. Set $u(t)=$ $\alpha(t)-y(t), t \in[a, b]$. Since $\alpha(w(t))-p(t, y(w(t))) \geq \min \{u(w(t)), 0\}, t \in I$, then by the definition of lower solution, we obtain

(i) $u^{(4)}(t)-M u(t)-N \min \{u(w(t)), 0\} \geq 0, \quad t \in I$,

(ii) $u^{(i)}(0)=u^{(i)}(T), \quad i=0,1,2$, $u^{(3)}(0) \geq u^{(3)}(T)$,

(iii) $u(0)=u(t), t \in[a, 0] \bigcup[T, b]$, 
(iv) $\frac{N}{M}\left(\frac{1}{\delta}-1\right)<1$, here $\delta=\frac{\left(e^{m T}-1\right) \cos \frac{m T}{2}+\left(e^{m T}+1\right) \sin \frac{m T}{2}}{e^{m T}+2 e^{\frac{m T}{2}} \sin \frac{m T}{2}-1}$.

Suppose, to the contrary, that $y(t)<\alpha(t)$ for some $t \in[0, T]$. It is enough to consider the following two cases.

Case 1: $u(t) \geq 0, u(t) \not \equiv 0$ on $I=[0, T]$.

In this case, we have that $u^{(3)}(0) \geq u^{(3)}(T)$ and $u^{(4)}(t) \geq 0, \quad t \in I$. Thus $u(t)=$ constant $=C>0$ on $I$, and we obtain

$$
0 \leq u^{(4)}(t)-M u(t)-N \min \{u(w(t)), 0\}=-M C
$$

which contradicts the fact that $C>0$.

Case 2: There exist $t_{1}, t_{2} \in[0, T]$ such that $u\left(t_{1}\right)>0$ and $u\left(t_{2}\right)<0$.

Let $u(\xi)=\min _{t \in[0, T]} u(t)=\min _{t \in[a, b]} u(t)<0$. Since $\min \{u(w(t)), 0\} \geq u(\xi)$, then

$$
u^{(4)}(t)-M u(t)-N u(\xi) \geq 0, \quad t \in[0, T] .
$$

As in the proof of Theorem 2.1 we obtain $u(t) \leq 0$. This implies $y \geq \alpha$. Similarly, we can prove $y \leq \beta$. Since $y \in[\alpha, \beta]$, this implies that $y$ is also a solution of $(3.1)$.

Finally, we will prove the uniqueness. Suppose that there exist two solutions $y_{1}$ and $y_{2}$ of (3.1) on $[\alpha, \beta]$. Applying Theorem 2.1 again one can prove $v=y_{1}-y_{2} \geq 0$ on $[a, b]$. As the same argument is valid for $y_{2}-y_{1}$, then $y_{2}-y_{1} \geq 0$. Thus $y_{1}=y_{2}$.

The proof of Theorem 3.1 is complete.

Now we are in a position to prove the validity of the monotone method for (1.1). First we shall introduce the concepts of lower and upper solutions for (1.1).

A function $\alpha \in E^{*}$ is said to be a lower solution to (1.1), if it satisfies

$$
\left\{\begin{array}{l}
\alpha^{(4)}(t) \geq f(t, \alpha(t), \alpha(w(t))), \quad t \in I \\
\alpha^{(i)}(0)=\alpha^{(i)}(T), \quad i=0,1,2, \\
\alpha^{(3)}(0) \geq \alpha^{(3)}(T) .
\end{array}\right.
$$

An upper solution for (1.1) is defined analogously by reversing the inequalities of above. A function $y \in E^{*}$ is said to be a solution to (1.1), if it is both an upper solution and a lower solution to (1.1).

THEOREM 3.2. Suppose that there exists a lower solution $\alpha$ and an upper solution $\beta$ of (1.1) such that $\alpha \leq \beta$ on $[a, b]$.

Assume that there exist constants $M=m^{4}, m \in(0, k), N>0$ satisfying

$\left(H_{1}\right) \quad f\left(t, u_{2}, v_{2}\right)-f\left(t, u_{1}, v_{1}\right) \leq M\left(u_{2}-u_{1}\right)+N\left(v_{2}-v_{1}\right)$, for $t \in I$, whenever $\alpha(t) \leq u_{1} \leq u_{2} \leq \beta(t)$ and $\alpha(w(t)) \leq v_{1} \leq v_{2} \leq \beta(w(t))$,

$\left(H_{2}\right) \quad \frac{N}{M}\left(\frac{1}{\delta}-1\right)<1$, here $\delta=\frac{\left(e^{m T}-1\right) \cos \frac{m T}{2}+\left(e^{m T}+1\right) \sin \frac{m T}{2}}{e^{m T}+2 e^{\frac{m T}{2}} \sin \frac{m T}{2}-1}$,

where $k$ is the smallest positive root of the equation $\tan \frac{m T}{2}=-\tanh \frac{m T}{2}$.

Then there exist two sequences $\left\{\alpha_{n}\right\}$ and $\left\{\beta_{n}\right\}$, nondecreasing and nonincreasing, respectively, with $\alpha_{0}=\alpha$ and $\beta_{0}=\beta$, which converge uniformly and monotonically to the extremal solution to the problem (1.1) in the segment $[\alpha, \beta]$. 
Proof. For each given $\eta \in[\alpha, \beta]$, we consider the PBVP (3.1) with

$$
\sigma(t)=\sigma_{\eta}(t)=f(t, \eta(t), \eta(w(t)))-M \eta(t)-N \eta(w(t)) .
$$

We shall refer to this problem as $(P L)_{\eta}$.

Since $\eta \in[\alpha, \beta]$ we have by $\left(H_{1}\right)$ and the definitions of lower and upper solutions, that

$$
\begin{aligned}
\alpha^{(4)}(t)-M \alpha(t) & -N \alpha(w(t)) \geq f(t, \alpha(t), \alpha(w(t)))-M \alpha(t)-N \alpha(w(t)) \\
& \geq f(t, \eta(t), \eta(w(t)))-M \eta(t)-N \eta(w(t))=\sigma_{\eta}(t)
\end{aligned}
$$

and

$$
\beta^{(4)}(t)-M \beta(t)-N \beta(w(t)) \leq \sigma_{\eta}(t) .
$$

As a consequence, $\alpha$ and $\beta$ are respectively a lower and an upper solution for $(P L)_{\eta}$ and Theorem 3.1 permits us to define the operator $A:[\alpha, \beta] \rightarrow[\alpha, \beta]$, where $A \eta$ is the unique solution of $(P L)_{\eta}$ on $[\alpha, \beta]$.

Concerning the mapping $A$, applying Theorem 3.1, it is easy to prove that

ClaIM 3.1. $A$ is a monotone increasing mapping on the segment $[\alpha, \beta]$, namely, $A \eta_{1} \leq A \eta_{2}$ when $\eta_{1}, \eta_{2} \in[\alpha, \beta]$ and $\eta_{1} \leq \eta_{2}$.

Thus we may define the sequences $\left\{\alpha_{n}\right\},\left\{\beta_{n}\right\}$ by $\alpha_{n+1}=A \alpha_{n}, \beta_{n+1}=A \beta_{n}$, $\alpha_{0}=\alpha, \beta_{0}=\beta$.

Using Claim 3.1 it is immediate that

$$
\alpha=\alpha_{0} \leq \alpha_{1} \leq \ldots \leq \alpha_{n} \leq \ldots \leq \beta_{n} \leq \ldots \leq \beta_{1} \leq \beta_{0}=\beta .
$$

Since $\left\{\alpha_{n}\right\}$ is nondecreasing, $\left\{\beta_{n}\right\}$ is nonincreasing, $\left\{\alpha_{n}^{(i)}\right\}$ and $\left\{\beta_{n}^{(i)}\right\}(i=0,1,2,3)$ are bounded in $C(I, R)$, we have that

$$
\lim _{n \rightarrow \infty} \alpha_{n}(t):=\alpha^{*}(t) \text { and } \lim _{n \rightarrow \infty} \beta_{n}(t):=\beta^{*}(t)
$$

uniformly and monotonically on $[a, b]$. Using the definition of $(P L)_{\eta}$ and passing the limit when $n$ tends to $\infty$, we conclude that $\alpha^{*}(t)$ and $\beta^{*}(t)$ are both solutions to the problem (1.1).

Furthermore, if $y \in[\alpha, \beta]$ is a solution to the problem (1.1), then,by induction, $\alpha_{n}(t) \leq y(t) \leq \beta_{n}(t)$ on $[a, b], n=0,1,2, \cdots$, and hence, $y \in\left[\alpha^{*}, \beta^{*}\right]$. This shows that $\alpha^{*}(t)$ and $\beta^{*}(t)$ are respectively minimal and maximal solutions to the problem (1.1) in the segment $[\alpha, \beta]$.

The proof of Theorem 3.2 is complete.

4. Monotone method for periodic solutions of FDEs. In this section, we are in a position to prove the validity of the monotone method for (1.2). First, we shall introduce the concepts of lower and upper solutions for these problems.

Let $X$ be defined as in Section 2 and let $X$ have the norm

$$
\|y\|_{4}=\max _{t \in[0, T]}\left(\sum_{i=0}^{4}\left|y^{(i)}(t)\right|\right)
$$

for $y \in X$. Then $X$ is a Banach space. 
A function $\alpha \in X$ is said to be a lower solution to (1.2), if it satisfies

$$
\alpha^{(4)}(t) \geq f(t, \alpha(t), \alpha(w(t))), \quad t \in R
$$

An upper solution for (1.2) is defined analogously by reversing the inequalities of above. A function $y \in X$ is said to be a solution to (1.2), if it is both an upper solution and a lower solution to (1.2).

By applying a similar argument as in Section 3, we can obtain the following result.

THEOREM 4.1. Suppose that there exists a lower solution $\alpha$ and an upper solution $\beta$ of (1.2) such that $\alpha \leq \beta$ in $R$.

Assume that there exist two constants $M=m^{4}, m \in(0, k), N>0$ satisfying

$\left(B_{1}\right) \quad f\left(t, u_{2}, v_{2}\right)-f\left(t, u_{1}, v_{1}\right) \leq M\left(u_{2}-u_{1}\right)+N\left(v_{2}-v_{1}\right)$, for $t \in R$, whenever $\alpha(t) \leq u_{1} \leq u_{2} \leq \beta(t)$ and $\alpha(w(t)) \leq v_{1} \leq v_{2} \leq \beta(w(t))$,

$\left(B_{2}\right) \frac{N}{M}\left(\frac{1}{\delta}-1\right)<1$, here $\delta=\frac{\left(e^{m T}-1\right) \cos \frac{m T}{2}+\left(e^{m T}+1\right) \sin \frac{m T}{2}}{e^{m T}+2 e^{\frac{m T}{2}} \sin \frac{m T}{2}-1}$.

where $k$ is the smallest root of the equation $\tan \frac{m T}{2}=-\tanh \frac{m T}{2}$.

Then there exist two sequences $\left\{\alpha_{n}\right\}$ and $\left\{\beta_{n}\right\}$, nondecreasing and nonincreasing, respectively, with $\alpha_{0}=\alpha$ and $\beta_{0}=\beta$, which converge uniformly and monotonically to the extremal $T$ - periodic solution to (1.2) in the segment $[\alpha, \beta]$.

\section{REFERENCES}

[1] G. S. Ladde, V. Lakshmikantham, and A.S. Vatsala, Monotone Iterative Techniques for Nonlinear Differential Equations, Pitman Advanced Publishing Program, 1985.

[2] S. Leela and M. N. Oguztoreli, Periodic boundary value problems for differential equations with delay and monotone iterative methods, J. Math. Anal. Appl., 122 (1987), pp. 301-307.

[3] J. R. Haddock and M. N. Nkashama, Periodic boundary value problems and monotone iterative methods for functional differential equations, Nonlinear Anal., 22 (1994), pp. $267-276$.

[4] E. Liz And J. J. Nieto, Periodic boundary value problems for a class of functional differential equations, J. Math. Anal. Appl., 200 (1996), pp. 680-686.

[5] J. J. Nieto, Differential inequalities for functional perturbations of first order differential equations, Applied Mathematics Letters, 15 (2002), pp. 173-179.

[6] D. JiAng, AND J. WeI, Monotone method for first- and second-order periodic boundary value problems and periodic solutions of functional differential equations, Nonlinear Anal., 50 (2002), pp. 885-898.

[7] A. CABADA AND S. LoIS, Maximum principles for fourth and sixth order periodic boundary value problems, Nonlinear Anal. , 29:10 (1997), pp. 1161-1171.

[8] A. CABADA, The method of lower and upper solutions for second, third, fourth and higher order boundary value problems, J. Math. Anal. Appl., 185 (1994), pp. 302-320.

[9] D. Jiang And L. Kong, A monotone method for constructing extremal solutions to secondorder periodic boundary value problems, Ann. Polon. Math., LXXVI (2001), pp. 279-285.

[10] D. JIANG, J.J. Nieto AND W. ZuO, On the monotone method for first and second order periodic boundary value problems and periodic solutions of functional differential equations, J. Math. Anal. Appl., 289 (2004), pp. 691-699.

[11] P.L. Buono and J. Belair, Restrictions and unfolding of double Hopf bifurcation in functional differential equations, J. Diff. Eqns., 189 (2003), pp. 234-266.

[12] T. Sengadir and A.K. Pani, Weak solutions of integro-differential and functional-differential equations, Diff. Eqns. Dynam. Sys., 4 (1996), pp. 411-422.

[13] T. TANigaWI, Oscillation and nonoscillation for a class of fourth order quasilinear functional differential equations, Hiroshima Math., 33 (2003), pp. 297-316.

[14] P.X. WenG, Upper and lower solutions method for the boundary value problem of a fourthorder functional differential equation (Chinese), J. South China Normal Univ. Natur. Sci. Ed., (2000), pp. 1-6.

[15] S. Zhang, X. Zheng And Z. Wang, Periodic solutions of a fourth order nonlinear differential equation, Soochow J. Math., 28 (2002), pp. 253-265. 
W. ZUO ET. AL. 